\title{
Prevention of Kinked Stent Graft Limb Due to Severe Angulated Proximal Neck during Endovascular Repair for Abdominal Aortic Aneurysm
}

\author{
Pyung Chun Oh, MD, Minsu Kim, MD, Eak Kyun Shin, MD, and Woong Chol Kang, MD
}

\begin{abstract}
Although the technology of endovascular aortic repair (EVAR) for abdominal aortic aneurysm (AAA) is evolving that make it appealing for challenging anatomy, proximal aortic neck morphology, especially severe angulation, is still one of the most determinants for a successful procedure. We describe a patient of AAA with severely angulated proximal neck, in whom kinked stent graft limb occurred against severe angulation of proximal neck. Then, we suggested how to prevent this complication in the second patient. Our case demonstrated the stent graft limb could be kinked by severe aortic neck angulation, making it challenging. However, the kinked stent graft limb could be prevented by deploying stent graft limbs below the most severely angulated aortic neck intentionally.
\end{abstract}

Keywords: AAA, EVAR, kinked stent graft limb

\section{Introduction}

Although the technology of endovascular aortic repair (EVAR) for abdominal aortic aneurysm (AAA) is evolving that make it appealing for challenging anatomy, proximal aortic neck morphology, especially severe angulation, is still one of the most determinants for a successful procedure. We describe a patient of AAA with severely angulated proximal neck, in whom kinked stent graft limb occurred against severe angulation of proximal neck during the EVAR. Then, we suggested how to prevent this complication in the second patient.

Department of Cardiology, Gachon University Gil Medical Center, Incheon, Korea

Received: April 4, 2017; Accepted: July 2, 2017

Corresponding author: Woong Chol Kang, MD. Department of Cardiology, Gachon University Gil Medical Center, 1198 Guwoldong, Namdong-gu, Incheon 405-760, Korea

Email: kangwch@gilhospital.com

(C)2018 The Editorial Committee of Annals of Thoracic and Cardiovascular Surgery. All rights reserved.

\section{Case 1}

An 87-year-old male patient presented with both leg edema. He had a history of hypertension, angina pectoris, and atrial fibrillation. Seven years ago, AAA had been incidentally found with a diameter of $43 \mathrm{~mm}$ and right internal iliac artery aneurysm $(38 \mathrm{~mm})$ had been treated with embolization using an Amplatzer Vascular Plug (St. Jude Medical, Plymouth, MN, USA). Then, he did not receive outpatient follow-up. At this time, AAA significantly increased to $75 \mathrm{~mm}$ in a diameter with a severely angulated proximal neck and concomitant aneurysm of both common iliac arteries at abdominal computed tomography (CT) (Figs. 1A and 1B). The angle between proximal neck and aneurysm was about $110^{\circ}$. For EVAR, the bifurcated stent graft (Endurant IIs, Medtronic Vascular, Santa Rosa, CA, USA) was deployed through the right common femoral artery. Then, we tried a cannulation of the contralateral limb of the bifurcated stent graft with 0.035 -inch hydrophilic guidewire (Terumo, Tokyo, Japan) via the left common femoral artery. However, the contralateral limb was completely kinked by ipsilateral limb due to severe angulation of 


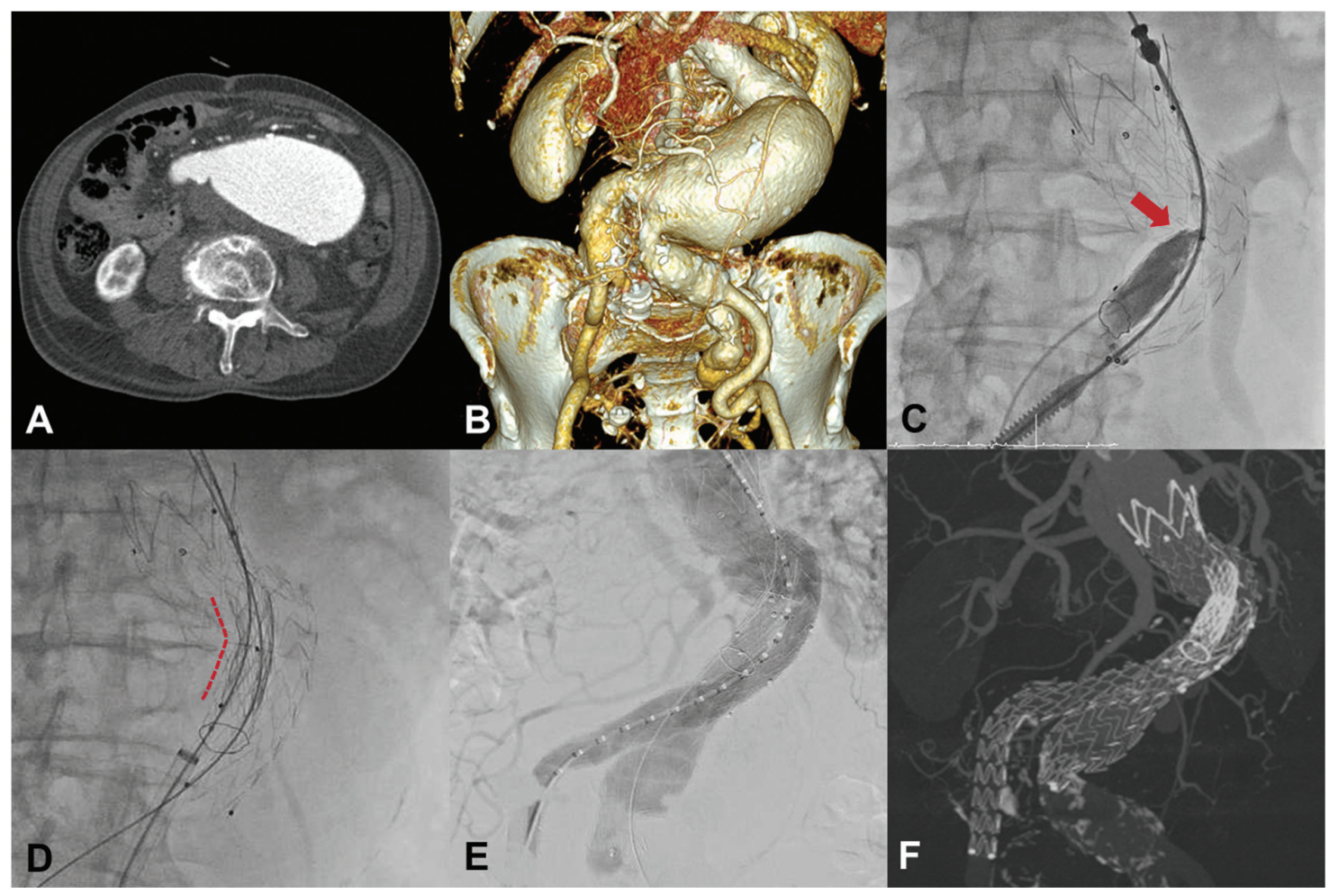

Fig. 1 (A, B) CT showed abdominal aorta aneurysm with severely angulated proximal aortic neck. (C) The contralateral limb was completely kinked (arrow) due to severe angulation of aortic neck. (D) A Palmaz stent (dotted line), manually mounted on a 12-mm diameter balloon, was implanted at the kinked limb. (E, F) Completion aortography and 2-month follow-up CT showed complete exclusion of aneurysm and patent stent grafts without kinking. CT: computed tomography

proximal neck and the guidewire could not be passed easily (Fig. 1C, arrow). After several attempts of wiring and manipulation of the knob of the bifurcated stent graft, we could pass the guidewire barely and changed the soft guidewire to extra stiff guidewire (Lunderquist, Cook Inc., Bloomington, IN, USA). Then, the kinked contralateral limb was dilated using an $8 \times 40 \mathrm{~mm}$ balloon catheter and a balloon expandable stent (Palmaz GENESIS, Cordis, Warren, NJ, USA), manually mounted on a $12-\mathrm{mm}$ diameter balloon, was implanted at the kinked contralateral limb (Fig. 1D, dotted line). The both iliac limb extension were placed to both common iliac arteries. Final aortography showed complete exclusion of aneurysm and patent stent grafts without kinking (Fig. 1E). After 2 months of the procedure, follow-up CT angiography demonstrated good patency of stent grafts without kinking of both limbs (Fig. 1F).

\section{Case 2}

Another 87-year-old male patient with a known AAA presented with dysarthria, which was diagnosed with acute cerebral infarction. He had a history of hypertension, diabetes mellitus, and angina pectoris. Abdominal CT angiography showed an AAA (67 $\mathrm{mm}$ in a diameter) with mural thrombus and concomitant aneurysm of both common iliac arteries (Figs. 2A and 2B). The proximal neck of the AAA was long and severely angulated in two parts, the mid-portion of proximal neck and the junction between proximal neck and aneurysmal sac.

From the lesson of the first case, the bifurcated portion of stent graft (Gore Excluder, WL Gore and Associates, Flagstaff, AZ, USA) (Fig. 2C, arrow) was intentionally deployed below the most angulated level (Fig. 2C, dotted line) of the mid-portion of proximal neck, which prevented kinking any limbs of the bifurcated stent grafts. Then, we implanted additionally a proximal aortic extender $(28.5 \times 33 \mathrm{~mm})$ for proximal sealing just below left renal artery (the lowest renal artery) (Fig. 2D, arrow). Both iliac limb extensions were placed to both common iliac arteries in a conventional manner. However, type III endoleak was noted at the junction between the proximal aortic extender and the bifurcated stent graft. So, an additional aortic extender 


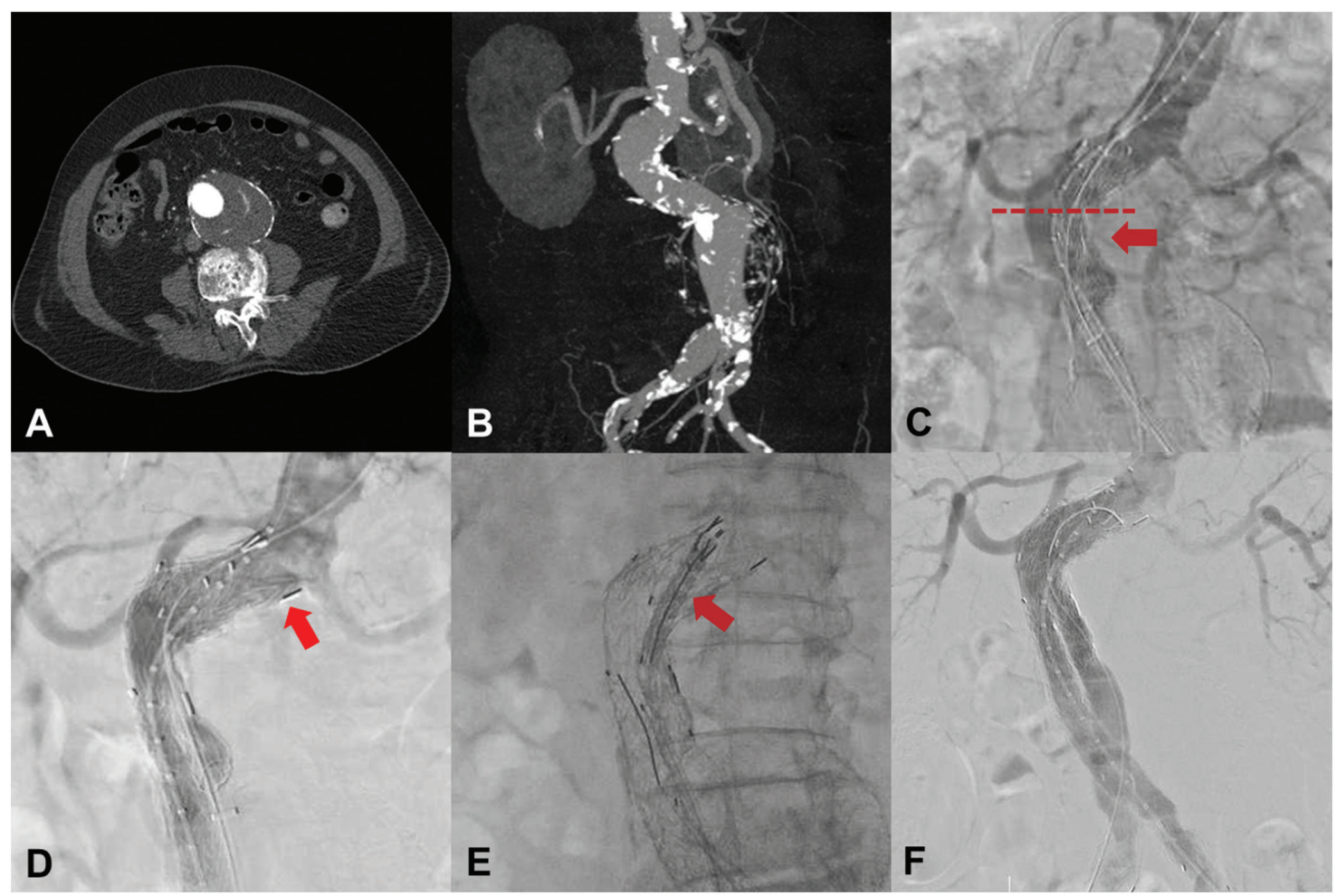

Fig. 2 (A, B) CT showed abdominal aorta aneurysm with severely angulation of proximal aortic neck. (C) The bifurcated portion of stent graft (arrow) was intentionally deployed below the most severely angulated level (dotted line) of the proximal neck. (D) Then, we implanted additionally a proximal aortic extender $(28.5 \times 33 \mathrm{~mm}$, arrow) for proximal sealing just below left renal artery (the lowest renal artery). (E) An aortic extender with larger diameter $(32 \times 45 \mathrm{~mm}$, arrow) was additionally implanted at the overlapping part of the proximal neck to treat type III endoleak. (F) Final aortography showed complete exclusion of aneurysm and good patency of stent grafts without kinking and endoleak. CT: computed tomography

with larger diameter $(32 \times 45 \mathrm{~mm})$ was implanted at the overlapping part of the proximal neck (Fig. 2E, arrow). Final aortography showed complete exclusion of aneurysm without any endoleak or kinked stent graft limb (Fig. 2F). After 1 month of the procedure, follow-up CT angiography demonstrated good patency of stent grafts without graft limb kinking and occlusion.

\section{Discussion}

Although EVAR is an established alternative to open repair for AAA, ${ }^{1)}$ an unfavorable aortic morphology is considered a major limiting factor for the successful procedure. Proximal aortic neck anatomy, especially severe angulation of proximal neck often complicates the procedure such as difficulties to easy access, accurate deployment, and proper fixation. Furthermore, the risks for late device-related complication including proximal type I endoleak or stent graft migration increase in patients with severe aortic neck angulation $\left.\left(>60^{\circ}\right)\right)^{2,3)}$
We also reported a case with kinked and thrombotic occlusion of the bifurcated stent graft limb due to severe angulation of aortic neck at 7 months after the EVAR. ${ }^{4)}$

In the first case, severely kinked stent graft limb at the side of lesser curvature of severe neck angulation occurred during the procedure, resulting in considerable technical difficulties. Before the procedure, we planned to implant both limbs of stent graft placed anteriorposterior relationship instead of side-by-side relationship to prevent kinking of stent graft. However, the direction of both limbs of stent graft could not be controlled by rotating the delivery system due to severe aortoiliac tortuosity. Although the technology of stent graft system is evolving to have highly conformable and kink-resistant features, commercially available devices are still limited for challenging anatomies, especially aortic neck angulation $>60^{\circ} .{ }^{5}$ )

In the second case, the mid-portion of proximal neck was more severely angulated than the junction between proximal neck and aneurysmal sac unlike in 
the first case. And the axial section of this part was elliptical (anterior-posterior diameter, $31 \mathrm{~mm}$; transverse diameter, $23 \mathrm{~mm}$ ), which might be prone to kink the limb of stent graft. Thus, we intentionally placed the each limb of bifurcated stent graft below the level of most severe angulation and added the proximal aortic extenders. Finally, kinked stent graft limb could be avoided by this approach. Although short-term outcomes of these cases were good, careful follow-up for late changes is mandatory.

\section{Conclusion}

Our cases demonstrated the stent graft limb could be kinked by severe aortic neck angulation during the procedure, making it challenging. Therefore, the expected location of each limb after stent graft deployment should be considered in patients with severe aortic neck angulation. The kinked stent graft limb could be prevented by deploying stent graft limbs below the most severely angulated aortic neck intentionally.

All procedures performed in our patients were in accordance with the ethical standards of the institutional and/or national research committee and with the 1964 Helsinki declaration and its later amendments or comparable ethical standards.

Informed consent was obtained from our patients.

\section{Disclosure Statement}

We declare that we have no conflicts of interest in connection with this paper. There is no fund for this study.

\section{References}

1) United Kingdom EVAR Trial Investigators, Greenhalgh RM, Brown LC, et al. Endovascular versus open repair of abdominal aortic aneurysm. N Engl J Med 2010; 362: 1863-71.

2) Sternbergh WC, Carter G, York JW, et al. Aortic neck angulation predicts adverse outcome with endovascular abdominal aortic aneurysm repair. J Vasc Surg 2002; 35: 482-6.

3) Hobo R, Kievit J, Leurs LJ, et al. Influence of severe infrarenal aortic neck angulation on complications at the proximal neck following endovascular AAA repair: a EUROSTAR study. J Endovasc Ther 2007; 14: 1-11.

4) Kim M, Kim MG, Kang WC, et al. Thrombotic occlusion of stent graft limbs due to severe angulation of aortic neck in endovascular repair of abdominal aortic aneurysm. Korean Circ J 2016; 46: 727-9.

5) Walker TG, Kalva SP, Yeddula K, et al. Clinical practice guidelines for endovascular abdominal aortic aneurysm repair: written by the standards of practice committee for the Society of interventional radiology and endorsed by the cardiovascular and interventional radiological Society of Europe and the Canadian Interventional radiology association. J Vasc Interv Radiol 2010; 21: 1632-55. 